\title{
Low Back Pain
}

by Weinstein and Gordon, American Academy of Orthopaedic Surgeons, 750 pp - Paperback - US \$ 83,95, ISBN 0-89203-160-3

This is a collective work, which gathers the works of a congress on New Horizons in Low Back Pain, which was held in November 1995 in SAN DIEGO California. The work is divided up into six sections including each ten chapters approximately.

The fist section concerns the epidemiology and the clinical translation of the lumbar radiculopathy. In this section it is recalled that the natural evolution of the discal hernia is favorable in approximately half the cases after approximately two weeks of evolution, that $70 \%$ of them recover after six weeks and this even with no surgery. The muscular exercises constitute an excellent prevention of the discal hernia. Because of the important anatomical differences which may exist in people without any symptomatology, the imaging must always be strongly correlated to the clinical signs. If the epidural corticosteroïd injections constitute a very popular treatment, it appears when one makes precise statistical studies that their efficiency is not at all proven. It is clear on the other hand that surgery after six weeks of clinical evolution gives good results. It is but difficult to show which type of surgery is the most efficient. To propose an arhtrodesis after the failure of a first discectomy treatment is only a small evidence, but on the other hand it is certain that this way of proceeding is good when a patient continues to suffer despite several discectomies. The discal surgery by arthroscopy takes more and more extent, but this technique has not been studied in a rigorous way.

The second section is dedicated to fundamental sciences concerning the lumbar radiculopathy. The mechanical effects of the discal hernia on the nerve root are not always clearly explainable. The volume of the discal hernia is not correlated to the severity of the pain. It appears that it would rather be the demyelinization ?? which would be responsible of this latter one. Other works showed that the discal hernia liberates cytokinases which would act on the nerve fibers.

Section three studies the epidemiology and the clinical translation of the idiopathic lumbalgia. One knows that the essential lumbalgia is extremely frequent within the adult population throughout the world. It has been advanced by some that this would be the consequence of the normal functioning of the vertebral column and not of pathologic traumatologic conditions. An extremely important question is to know whether the anatomic modifications are effectively responsible for pains or if there are asymptomatic degenerations. Certain notions are well established. One knows thus that subjects realizing important efforts of uplifts have a greater frequency of lumbalgix than sedentaries. The role of skeletal muscles is on the other hand subject to controversy. If reeducation exercises and other means of physiotherapy have a very important place in the treatment of the essential lumbalgia, their objective justification on the other hand is difficult to prove. Similarly the manipulations are certainly useful in case of acute lumbalgiæ, but it is not proven that they would be efficient in the chronic lumbalgia.
Section 4 concerns the place of fundamental sciences in the essential lumbalgia. After a reminder on the functioning of collagen and the discal innervation, the study is interested in the biomechanical behavior of the intervertebral disk. This functioning is complex. the normal nucleus pulposus functions like a fluid placed under a static compression. The strong pression due to the concentration of the glycoproteins in the nucleus maintains the discal height and contributes to fight against the charge of the on each disk. The hydraulic permeability of the cartilage and of the vertebral plate acts to evenly distribute this load in a uniform way towards the annulus fibrosus and the nucleus pulposus.

The different animal models allow to assert that the discal degenerescence contributes to the common lumbalgia according to two mechanisms. The first mechanism is the loss of discal height which echoes on the posterior articular facets. The second factor is the liberation of cytokinase and free radicals, of prostaglandins and other mediators which have a nociceptive effect on the nerve ends.

The fifth chapter is dedicated to the epidemiology and the clinical result of the lumbar stenosis. The natural history of the lumbar stenosis is not well known, but it appears that one is satisfied with a medical treatment even in case degenerative spondylolisthesis, the increase of the deterioration occurs only in ten per cent of the cases and that on the other hand a third of the patients are ameliorated. The non surgical treatment too was not the object of important studies. The epidural injections did not make their proofs and are often proposed. The majority of patients are treated by antalgics, a lumbar orthesis, and reeducation, but here again there is no serious study known. This should be the object in a priority way of an epidemiologic study.

The surgical treatment suffers also from a lack of randomization. One knows that the range of the laminectomy is matter to debate. Should one arthrodese at the same time ? Here again there are to few examples in order to draw conclusions. Some elements are nevertheless known, that is to say that the existence of an important comorbidity gives in general bad results, that the predominance of radicular pains gives better results only if one grapples with the unique lumbalgia.

This work is very agreeable to consult, as each section is preceded by an overview which allows then a deepen reading of the chapter which will interest most the reader. The work is of course completed by an index, which allows more targeted entries. This work is intended to all those who are concerned with the lumbalgia, the Rheumatologists, the orthopædic Surgeons and NeuroSurgeons, the Physiotherapists and the Fundamentalists interested by the the intervertebral disk.

\section{Erratum}

P. Harrington, F. McGrath, R. Moran (1998) Magnetic resonance imaging of the extremities. A critical appraisal of utilization by an orthopaedic service. Eur J Orthop Surg Traumatol 8: 157-160

Figure 3 on page 163 should be turned $180^{\circ}$ for correct viewing. 